\title{
Creatine Deficiency in the Brain: A New, Treatable Inborn Error of Metabolism
}

\author{
S. STÖCKLER, U. HOLZBACH, F. HANEFELD, I. MARQUARDT, G. HELMS, \\ M. REQUART, W. HÄNICKE, AND J. FRAHM
}

Kinderklinik, Universität Göttingen, Germany [S.S., U.H., F.H.]; Kinderklinik, Städtische

Kliniken Oldenburg, Germany [I.M.]; Biomedizinische NMR Forschungs GmbH, Max-Planck-Institut für biophysikalische Chemie, Göttingen, Germany [G.H., M.R., W.H., J.F.]

\section{ABSTRACT}

\begin{abstract}
In a patient with extrapyramidal movement disorder and extremely low creatinine concentrations in serum and urine, in vivo proton magnetic resonance spectroscopy disclosed a generalized depletion of creatine in the brain. Oral substitution of arginine, a substrate for creatine synthesis, resulted in an increase of brain guanidinoacetate as the immediate precursor of creatine but did not elevate cerebral creatine levels. In contrast, oral substitution of creatine-monohydrate led to a significant increase of brain creatine, a decrease of brain guanidinoacetate, and a normalization of creatinine in serum and urine. Phosphorus
\end{abstract}

magnetic resonance spectroscopy of the brain revealed no detectable creatine-phosphate before oral substitution of creatine and a significant increase afterward. Partial restoration of cerebral creatine concentrations was accompanied by improvement of the patient's neurologic symptoms. This is the first report of a patient with complete creatine deficiency in the brain. Magnetic resonance spectroscopy during arginine and creatine treatment point to an inborn error of creatine biosynthesis at the level of guanidinoacetete-methyltransferase. (Pediatr Res 36: 409-413, 1994)
Creatine ( $\alpha$-methyl-guanidinoacetic acid) and creatinephosphate play essential roles in the storage and transmission of phosphate-bound energy. In humans, creatine is synthesized in liver and pancreas using arginine, glycine, and S-adenosyl-methionine as substrates and arginine:glycine amidinotransferase and guanidinoacetatemethyltransferase as enzymes (Fig. 1). Creatine is not utilized in these organs of synthesis but transported to muscle and nerve tissue, which contain high activities of creatine kinase. This enzyme catalyzes the phosphorylation and dephosphorylation of creatine and creatinephosphate and thus provides a high-energy phosphatebuffering system during states of ATP synthesis and ATP utilization. Creatine and creatine-phosphate are converted at constant fractional rates to creatinine, which is excreted in urine. In humans, the creatine/creatinephosphate pool is maintained by endogenous biosynthesis and by nutritional intake of creatine (1-3).

In vivo proton and phosphorus magnetic resonance spectroscopy have been used for a noninvasive assessment of amino acid and energy metabolism including the creatine/creatine-phosphate pool in brain and muscle (4,

Received for rapid publication April 5, 1994; accepted June 7, 1994.

Correspondence and reprint requests: Prof. Dr. Folker Hanefeld, Univ. Kinderklinik/Schwerpunkt Neuropädiatrie, Robert-Koch-Straße 40, D-37075 Göttingen, Germany.
5). So far, extremely low creatine levels have only been observed in brain tumors such as gliomas, astrocytomas, and meningiomas (6) as well as in three children with an encephalopathy leading to severe structural and metabolic alterations in white matter (7). This is the first report of a patient with a selective deficiency of creatine and creatine-phosphate in the brain. The findings by magnetic resonance spectroscopy strongly suggest a previously unknown inborn error of creatine biosynthesis.

\section{CASE REPORT}

This male patient was born after an uncomplicated pregnancy and delivery to healthy, unrelated German parents. Psychomotor development was considered normal until 5 mo of age. After a developmental arrest, he gradually developed a severe extrapyramidal disorder. At the age of $22 \mathrm{mo}$, he was severely hypotonic and unable to sit or roll over. Uncoordinated swallowing and frequent vomiting required transient nasogastric tube feeding. His arm and hand movements were of a particular almost hemiballistic-dystonic type. In a sitting position, constant head dropping could be observed. His head circumference, hearing, and vision appeared normal. There was no organomegaly.

Normal values were obtained for blood cell count, blood gas analysis, serum electrolytes, glucose, urea, 


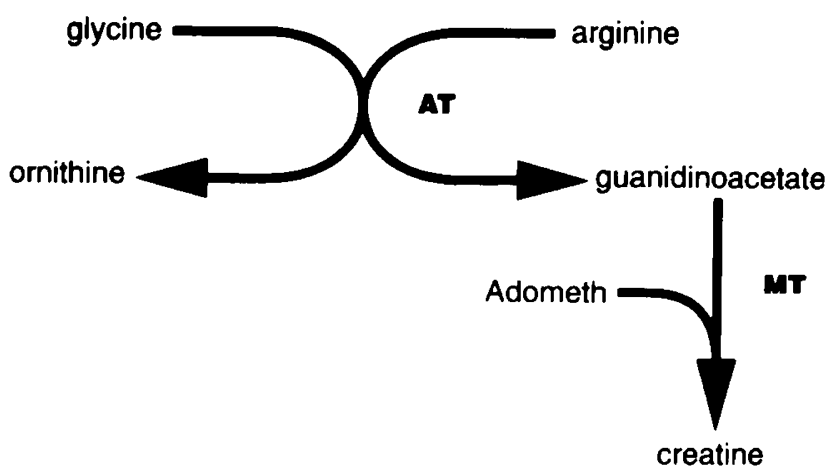

Figure 1. Biosynthesis of creatine in human liver and pancreas involves arginine:glycine amidinotransferase $(A T)$ and guanidinoacetate methyltransferase $(M T)$ as enzymes and glycine, arginine, and S-adenosylmethionine (Adometh) as substrates.

uric acid, cholesterol, triglycerides, liver aminotransferases, creatine kinase, lactate dehydrogenase, alkaline phosphatase, and blood coagulation tests. Cerebrospinal fluid was normal for cell count, protein, glucose, and lactic acid. A cytogenetic investigation of the chromosomes revealed a normal karyotype $(46, \mathrm{XY})$. ECG and cardiac ultrasound examination were normal. The EEG showed a very slow background activity $(1.5-3 / \mathrm{s})$ and multifocal spike slow waves. Cranial magnetic resonance imaging revealed bilateral abnormalities of the globus pallidus as hypointensities in T1-weighted images and as hyperintensities in T2-weighted images.

Particular metabolic findings included hyperammonemia $(100-120 \mu \mathrm{mol} / \mathrm{L}$; normal, $10-70 \mu \mathrm{mol} / \mathrm{L})$, orotic aciduria $(27-29 \mathrm{mmol} / \mathrm{mol}$ creatinine; normal, $0.1-0.5$ $\mathrm{mmol} / \mathrm{mol})$, and hyperornithinemia $(200-400 \mathrm{mmol} / \mathrm{L}$; normal, $10-110 \mathrm{mmol} / \mathrm{L}$ ) in the presence of low plasma arginine concentrations $(5-15 \mathrm{mmol} / \mathrm{L}$; normal, $10-65$ $\mathrm{mmol} / \mathrm{L})$. Urinary excretion of dicarboxylic acids, 3-methylglutaconic acid, and ethylmalonic acid was elevated in relation to extremely low urinary creatinine concentrations $(90-700 \mu \mathrm{mol} / \mathrm{L}$; normal, $1800-4400 \mu \mathrm{mol} / \mathrm{L})$. Serum creatinine was unusually low as well (7-10 $\mu \mathrm{mol} / \mathrm{L}$; normal, $25-100 \mu \mathrm{mol} / \mathrm{L})$. Urea cycle defects and the hyperammonemia-hyperornithemia-homocitrullinuria syndrome (8) were excluded by an otherwise normal pattern of plasma and urinary amino acids. In particular, urinary homocitrulline was not detectable on several occasions and hyperammonemia normalized without a specific treatment. Fatty acid oxidation defects were excluded by normal oxidation rates of myristic acid and palmitic acid in fibroblasts. Mitochondrial respiratory chain and pyruvate dehydrogenase defects were excluded by normal activities of these enzymes in muscle tissue.

Because the aforementioned results remained inconclusive, the patient received mild sedation and his condition was investigated by magnetic resonance spectroscopy. Written informed consent was obtained from the parents before these examinations. Image-controlled localized proton magnetic resonance spectroscopy was performed at 2.0 Tesla (Magnetom SP4000, Siemens,
Erlangen, Germany) using a short-echo time STEAM sequence as described previously (9). Quantification of absolute metabolite concentrations was based on fully relaxed spectroscopic acquisitions (i.e. repetition times TR $=6000 \mathrm{~ms}$ ) and involved user-independent automated spectral evaluation (10) with use of a library of calibrated spectra from model metabolite solutions (11). Phosphorus magnetic resonance spectroscopy was carried out without localization using a circumscribing phosphorus head coil.

Proton magnetic resonance spectroscopy revealed a complete deficiency of creatine in gray and white matter, basal ganglia, and cerebellum. Typical spectra of gray and white matter are shown in Figure 2. In addition to resonances from $\mathrm{N}$-acetylaspartate, choline-containing compounds, and myo-inositol, the spectra exhibited a new resonance that became even more prominent after a 4-wk oral substitution of $\mathrm{L}$-arginine $(300 \mathrm{mg} / \mathrm{kg} / \mathrm{d}$, Ajinomoto, Hamburg, Germany) as demonstrated by a comparison of the upper two traces in Figure 2. No other alterations of metabolite levels were observed during arginine substitution, with creatine remaining undetectable. Identification of the new resonance as guanidinoacetate was accomplished by using model metabolite solutions both at high magnetic fields and at 2.0 Tesla under experimental conditions identical with those used for in vivo studies (unpublished results).

Six wk of oral substitution of $\mathrm{L}$-arginine $(150 \mathrm{mg} / \mathrm{kg} / \mathrm{d})$ and creatine-monohydrate $(400 \mathrm{mg} / \mathrm{kg} / \mathrm{d}$, Merck, Darmstadt, Germany) led to an impressive increase of creatine in both gray and white matter (Fig. 2, third row of traces). The concentration of guanidinoacetate remained elevated. After an additional 6-wk substitution of creatinemonohydrate $(400 \mathrm{mg} / \mathrm{kg} / \mathrm{d})$ without $\mathrm{L}$-arginine, the brain creatine had reached almost $50 \%$ of its normal concentration, whereas guanidinoacetate was reduced to normal low values (Fig. 2, bottom traces). Table 1 summarizes quantitative alterations of concentrations for major metabolites during the course of consecutive therapeutic regimens.

The findings with phosphorus magnetic resonance spectroscopy paralleled those obtained with proton spectroscopy. During oral substitution of arginine, no creatine-phosphate was detectable. Instead, phosphorus spectroscopy revealed a strong resonance due to guanidinoacetate-phosphate that is distinct from the phosphate resonance of creatine-phoshate. After oral substitution of creatine-monohydrate, guanidinoacetate-phosphate nearly vanished over the course of $12 \mathrm{wk}$ strictly complementary to an increase of creatine-phosphate (unpublished results).

There was no clinical improvement during substitution of L-arginine, with serum and urinary creatinine concentrations still extremely low. Substitution of creatinemonohydrate, however, resulted in a significant improvement of muscle tone and extrapyramidal symptoms. The boy became more alert, followed with his eyes, grasped and moved small toys from one hand to the other, and began to turn over and to crawl. The EEG returned to a regular theta activity (4-6/s). In cranial magnetic reso- 


\section{Gray Matter}
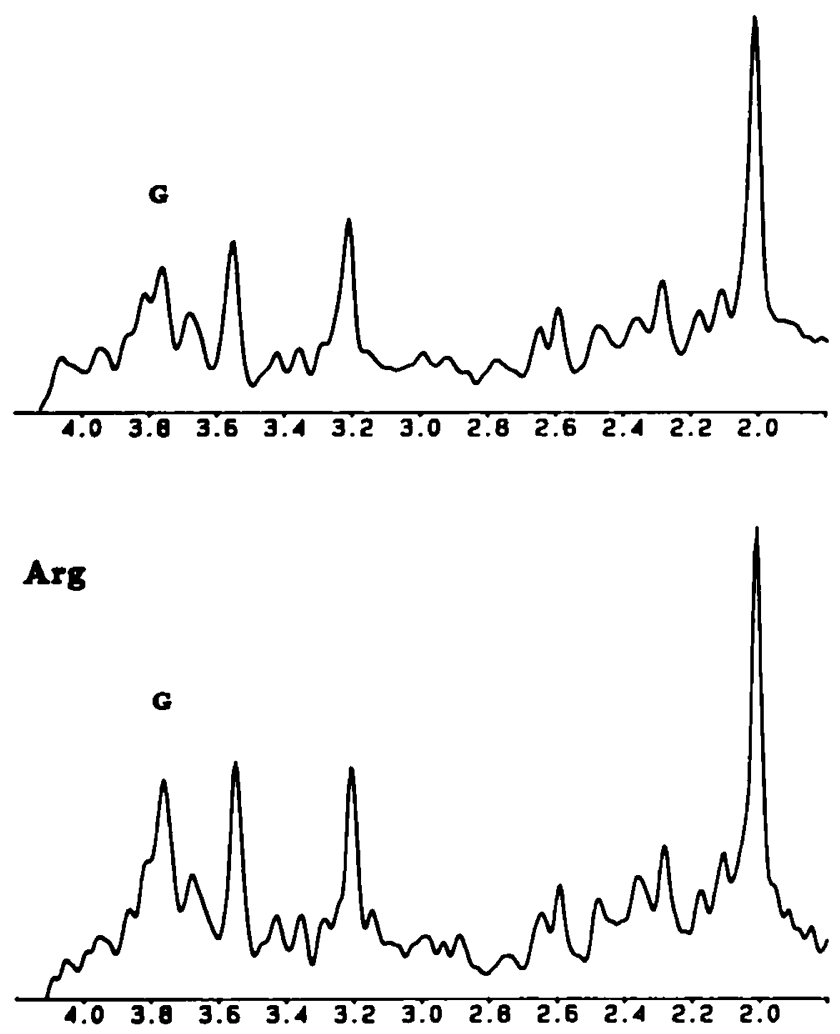

ArgtCr
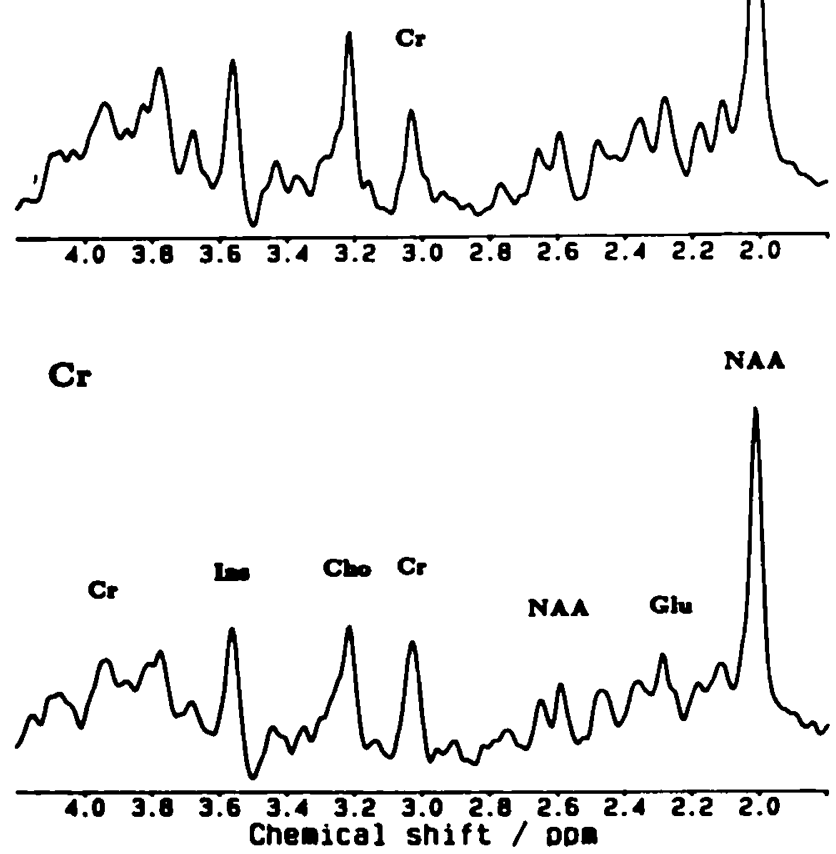

White Matter
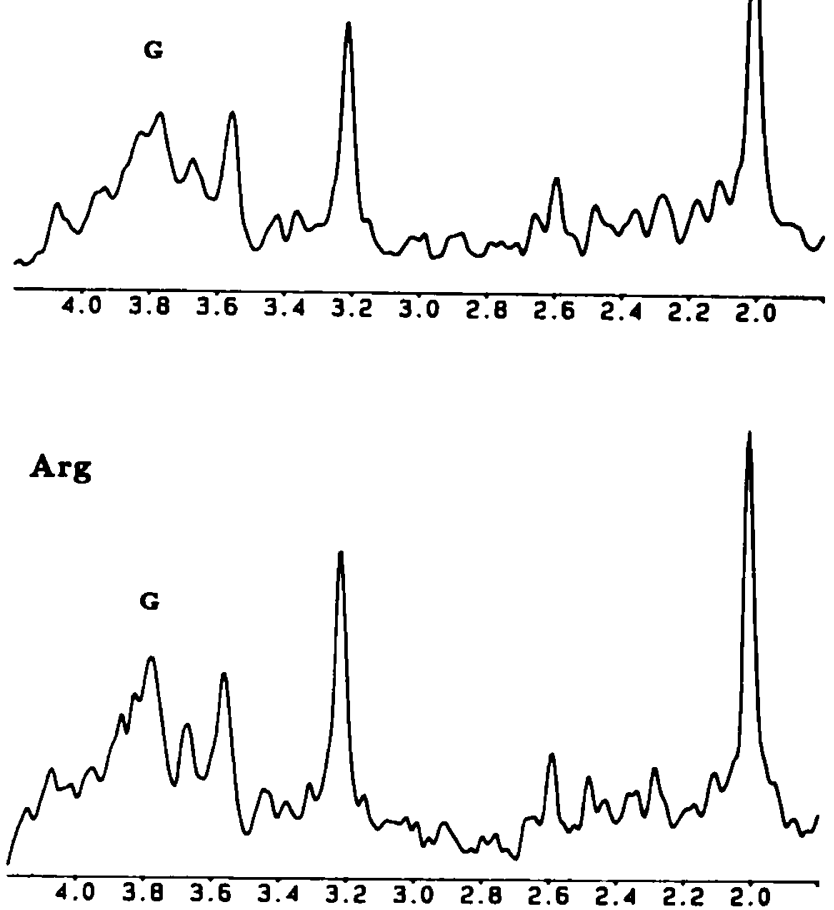

Figure 2. Localized proton magnetic resonance spectra of parietal gray matter (left: $18 \mathrm{~mL}$ volume) and white matter (right: $7.7 \mathrm{~mL}$ volume) in a patient with complete creatine deficiency (top traces), in the patient at $22 \mathrm{mo}$ of age after a 4-wk substitution of L-arginine (second row of traces), and after a 6-wk substitution of creatine (bottom traces). Resonance assignments are due to $N$-acetylaspartate $(N A A)$, glutamate (Glu), creatine and creatine-phosphate $(\mathrm{Cr})$, choline-containing compounds $(\mathrm{Cho})$, myoinositol $(\mathrm{Ins})$, and guanidinoacetate $(G)$. 
Table 1. Absolute concentrations ( $\mathrm{mmol} / \mathrm{L})$ of $\mathrm{N}$-acetylaspartate $(\mathrm{NAA})$, creatine and creatine-phosphate (Cr), choline-containing compounds (Cho), myo-inositol (Ins), and guanidinoacetate $(G)$ in gray and white matter of a patient with creatine deficiency*

\begin{tabular}{|c|c|c|c|c|c|c|}
\hline & NAA & $\mathrm{Cr}$ & Cho & Ins & G & \\
\hline Gray matter & 6.2 & 0.2 & 1.0 & 4.1 & 3.6 & $22 \mathrm{mo}$ \\
\hline Arg & 5.9 & 0.1 & 1.1 & 4.0 & 8.6 & $23 \mathrm{mo}$ \\
\hline $\mathrm{Arg}+\mathrm{Cr}$ & 6.4 & 1.8 & 1.1 & 3.7 & 6.0 & $25 \mathrm{mo}$ \\
\hline $\mathrm{Cr}$ & 6.0 & 2.6 & 0.9 & 4.1 & $(2.0)$ & $26 \mathrm{mo}$ \\
\hline Control $(n=27)$ & $7.3 \pm 1.3$ & $5.5 \pm 0.8$ & $1.0 \pm 0.2$ & $3.7 \pm 0.7$ & $(1.6 \pm 1.0)$ & \\
\hline White matter & 6.6 & 0.3 & 1.6 & 3.6 & 3.4 & $22 \mathrm{mo}$ \\
\hline Arg & 6.7 & 0.4 & 1.7 & 3.4 & 8.0 & $23 \mathrm{mo}$ \\
\hline \multirow[t]{2}{*}{$\mathrm{Arg}+\mathrm{Cr}$} & L: 7.1 & 1.1 & 1.8 & 2.6 & 6.8 & $25 \mathrm{mo}$ \\
\hline & $\mathrm{R}: 7.3$ & 1.3 & 1.8 & 1.8 & $(2.0)$ & \\
\hline \multirow[t]{2}{*}{$\mathrm{Cr}$} & L: 6.9 & 1.9 & 1.6 & 1.9 & $(0.6)$ & $26 \mathrm{mo}$ \\
\hline & $\mathrm{R}: 6.9$ & 1.9 & 1.8 & 1.9 & (2.3) & \\
\hline Control $(n=46)$ & $8.9 \pm 1.5$ & $5.1 \pm 0.9$ & $1.5 \pm 0.3$ & $3.2 \pm 0.9$ & $(0.9 \pm 0.9)$ & \\
\hline
\end{tabular}

* Controls are from young adults $(26 \pm 3 \mathrm{y})$ because no age-matched data were available. Guanidinoacetate levels below $3 \mathrm{mmol} / \mathrm{L}$ are of low significance (values given in parentheses). L, left; $R$, right.

nance imaging, the abnormal signal intensities of the globus pallidus became less pronounced. Serum and urinary creatinine concentrations normalized $(27$ and 1884 $\mu \mathrm{mol} / \mathrm{L})$ as did urinary orotic acid excretion $(0.2-0.4$ $\mathrm{mmol} / \mathrm{mol}$ creatinine), and serum ornithine concentrations $(60 \mathrm{mmol} / \mathrm{L})$.

\section{DISCUSSION}

In this patient suffering from a severe extrapyramidal disorder, extremely low serum and urinary creatinine concentrations were apparent in addition to a number of inconsequential metabolic disturbances. In vivo magnetic resonance spectroscopy disclosed a generalized depletion of cerebral creatine (and creatine-phoshate) and elucidated the metabolic background by monitoring the time course of metabolite concentrations in response to oral supplementation of arginine and creatine. The identification of guanidinoacetate as a new substance contributing to proton (and in its phosphorylated form to phosphorus) spectra allowed definition of the underlying metabolic defect. The significant increase of cerebral guanidinoacetate without a simultaneous increase of brain creatine after substitution of L-arginine indicates that the fist step of creatine biosynthesis catalyzed by arginine:glycine amidinotransferase is unaffected, whereas methylation of guanidinoacetate as the final step in creatine biosynthesis is defective. Obviously, the metabolic block in the patient is due to an inborn error of guanidinoacetate-methyltransferase. Measurements of this enzyme are under way.

Creatine effects the regulation of arginine:glycine amidinotransferase (3): In the presence of creatine, arginine:glycine amidinotransferase is repressed. In this patient, an abnormal derepression of arginine:glycine amidinotransferase in the creatine-deficient state and a normal repression in the creatine-supplemented state might explain the high brain guanidinoacetate levels before creatine substitution as well as the significant decrease thereafter. Because guanidinoacetate and orni- thine are produced in equimolar amounts from arginine and glycine by arginine:glycine amidinotransferase, the same might be true for the high plasma ornithine and low arginine concentrations initially found.

The normal neurologic development during the first few months of life suggests that this patient might have been fully provided with maternal creatine in utero and that it took some weeks after birth to become depleted of the maternal creatine pool. A similar time course was observed for brain creatine restoration during oral substitution, which resulted in about $50 \%$ of normal brain creatine after $12 \mathrm{wk}$. The concomitant increase of brain creatine-phosphate as measured by phosphorus spectroscopy indicates a normal activity of creatine kinase in the brain and therefore a normal metabolic function of the substituted creatine. Moreover, the rise of plasma and urinary creatinine concentrations underlines normal degradation of a proportion of the creatine/creatinephosphate pool.

In conclusion, it is not yet clear whether the neurologic symptoms observed in this patient result from an imbalance of high-energy phosphates or from direct neurotoxicity of guanidinoacetate. Nevertheless, the striking improvement of this patient's clinical symptoms during creatine substitution suggests that early recognition and treatment of creatine deficiency might largely prevent brain injury. Further experience with this new inborn error of metabolism and a longer follow-up under creatine substitution are required to answer this question. The disease described here may serve as an important model for studying cerebral maturation, function, and dysfunction.

Acknowledgments. The authors thank Dr. C. Bachmann, Lausanne, Switzerland, and Dr. W. Lehnert, Freiburg, Germany, for determination of urinary organic acids; Dr. D. Byrd, Hannover, Germany, for determination of urinary orotic acid and homocitrulline; Dr. W. Ruitenbeek, Nijmegen, Netherlands, for determination 
of mitochondrial respiratory chain enzymes and pyruvate dehydrogenase in muscle tissue; and Dr. E. Christensen, Copenhagen, Denmark, for determination of fatty acid oxidation rates in fibroblasts.

\section{REFERENCES}

1. Hochachka PW 1985 Fuels and pathways as designed for support of muscle work. J Exp Biol 115:149-164

2. Walker JB 1963 Formamindine group transfer in extracts of human pancreas. liver, and kidney. Biochim Biophys Acta 73:241-247

3. Walker JB 1979 Creatine: biosynthesis, regulation, and function. Adv Enzymol 50:177-242

4. Radda GK 1986 The use of NMR spectroscopy for the understanding of disease. Science 233:640-645

5. Howe FA, Maxwell RJ, Saunders DE, Brown MM, Griffiths JR 1993 Proton spectroscopy in vivo. Magn Reson Q 9:31-59
6. Frahm J, Bruhn H, Merboldt KD, Hänicke W, Mursch D, Markakis E 1991 Localized proton NMR spectroscopy of brain tumors. Methodologic improvements using short-echo time STEAM sequences. J Comput Assist Tomogr 15:915-922

7. Hanefeld F, Holzbach U, Kruse B, Wilichowski E, Christen HJ, Frahm J 199.3 Diffuse white matter disease in three children: an encephalopathy with unique features on magnetic resonance imaging and proton magnetic resonance spectroscopy. Neuropediatrics $24: 244-248$

8. Valle D, Simell O 1989 The hyperornithinemias. In: Scriver CR, Beaudet AL. Sly SW, Valle D (eds) The Metabolic Basis of Inherited Disease, 6th Ed. McGraw-Hill, New York, pp 599-627

9. Frahm J, Michaelis T, Merboldt, KD, Bruhn H, Gyngell ML, Hänicke W 1990 Improvements in localized proton NMR spectroscopy of human brain. Water suppression, short echo times, and $1 \mathrm{~mL}$ resolution. J Magn Reson 9):464-473

10. Provencer $S W 1993$ Estimation of metabolite concentrations from localized in vivo proton NMR spectra. Magn Reson Med 30:672-679

11. Michaelis T, Merboldt KD, Bruhn H, Hänicke W, Frahm J 1993 Absolute concentrations of metabolites in the adult human brain in vivo. Quantification of localized proton MR spectra. Radiology 187:219-227 